
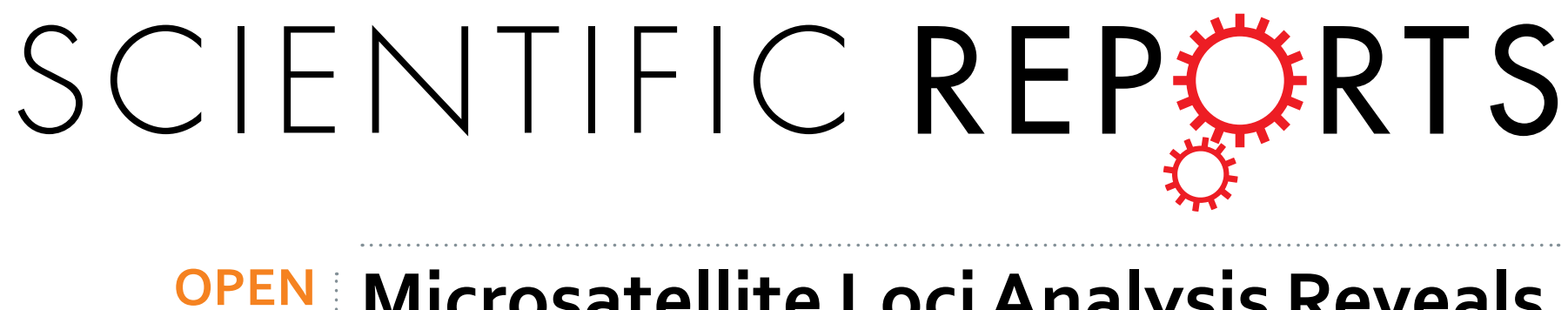

\title{
Microsatellite Loci Analysis Reveals Post-bottleneck Recovery of Genetic Diversity in the Tibetan \\ Antelope
}

Received: 27 May 2016

Accepted: 30 September 2016

Published: 14 October 2016

\author{
Yurong Du ${ }^{1}$, Xiaoyan Zou ${ }^{2}$, Yongtao Xu ${ }^{3}$, Xinyi Guo ${ }^{3}$, Shuang $\mathrm{Li}^{1}$, Xuze Zhang ${ }^{4}$, Mengyu Su${ }^{1}$, \\ Jianbin $\mathrm{Ma}^{1}$ \& Songchang $\mathrm{Guo}^{2}$
}

The Tibetan antelope (chiru, Pantholops hodgsoni) is one of the most endangered mammals native to the Qinghai-Tibetan Plateau. The population size has rapidly declined over the last century due to illegal hunting and habitat damage. In the past 10 years, the population has reportedly been expanding due to conservation efforts. Several lines of evidence suggest that the Tibetan antelope has undergone a demographic bottleneck. However, the consequences of the bottleneck on genetic diversity and the post-bottleneck genetic recovery remain unknown. In this study, we investigate the genetic variation of 15 microsatellite loci from two Tibetan antelope populations sampled in 2003 (Pop2003) and 2013 (Pop2013). A higher level of genetic diversity (NA, 13.286; He, $0.840 ;$ PIC, $0.813 ; I, 2.114$ ) was detected in Pop2013, compared to Pop2003 (NA, 12.929; He, 0.818; PIC, 0.789; I, 2.033). We observe that despite passing through the bottleneck, the Tibetan antelope retains high levels of genetic diversity. Furthermore, our results show significant or near significant increases in genetic diversity ( $\mathrm{He}, \mathrm{PIC}$ and $I$ ) in Pop2013 compared with Pop2003, which suggests that protection efforts did not arrive too late for the Tibetan antelope.

The Tibetan antelope or chiru (Pantholops hodgsonii) is a member of the Bovidae family (order Artiodactyla) native to the Qinghai-Tibet Plateau (QTP). It is distributed primarily throughout central Tibet, Qinghai, Xinjiang and west Sichuan in China, occupying a total area of approximately $880,000 \mathrm{~km}^{2}$ at an altitude of 3700 to $5500 \mathrm{~m}^{1}$. The Tibetan antelope was widely distributed over the QTP in the $20^{\text {th }}$ century, with a population size ranging from 500,000 to $1,000,000$ individuals during peak years ${ }^{2}$. However, this species has suffered a severe demographic bottleneck since the 1950s. The estimation of Tibetan antelope population size reached the minimum number of 50,000 individuals in $2003^{3}$. Such a drastic reduction is primarily considered to be the result of human activity such as poaching and overgrazing. Accordingly, the Tibetan antelope is listed as "Endangered" on the IUCN Red List (http://www.iucnredlist.org/details/15967/0) and as a Category I species under the Wild Animal Protection Law of China. To protect the Tibetan antelope and restore the population, the Chinese government has set up seven Nature Reserves since $1993^{4}$ and has taken measures such as anti-poaching, building wildlife passageways, and strengthening publicity. In 2011, the number of Tibetan antelope was estimated to have recovered up to 200,000 (Antelope Specialist Group 2011, See ref. 3).

Nevertheless, Tibetan antelope populations are still suffering from pressure from poaching, the development of society by man, and the worsening environment as a result of global warming and grassland degradation caused by human activities. Genetic variant analysis based on SSR and mtDNA markers in the giant panda ${ }^{5}$, grey shrike ${ }^{6}$ and Sichuan snub-nosed monkey ${ }^{7}$ suggest that declines in population lead to the rapid loss of genetic diversity and, thus, the danger of population extinction. Although the Tibetan antelope population continues to grow, it is unclear whether the genetic diversity of this species is increasing over time. Furthermore, the recovery of endangered species from severe population bottlenecks now frequently involves human intervention, but the

${ }^{1}$ School of Life and Geography Science, Qinghai Normal University, Xining 810008, China. ${ }^{2}$ Key Laboratory of Evolution and Adaptation of Plateau Biota, Northwest Institute of Plateau Biology, Chinese Academy of Sciences, Xining 810007, China. ${ }^{3}$ School of Life Science, Sichuan University, Chengdu 610064, China. ${ }^{4}$ School of Chemistry and Chemical Engineering, Qinghai University for Nationalities, Xining 810001, China. Correspondence and requests for materials should be addressed to J.M. (email: mjb_117@163.com) or S.G. (email: guo@nwipb.cas.cn) 


\begin{tabular}{|c|c|c|c|}
\hline Locus & Primers & Core sequence & $\mathrm{Ta}^{\circ} \mathrm{C}$ \\
\hline \multirow{2}{*}{ P1 } & F: AGCAAGCATTTGTCTGTCAGT & \multirow{2}{*}{ (AC) $12(\mathrm{CA}) 14$} & \multirow{2}{*}{52.8} \\
\hline & R: GTATGGCAGGTGGGGAAT & & \\
\hline \multirow{2}{*}{ P6 } & F: TGATCCATAAAACCAGGGGA & \multirow{2}{*}{ (GT) 14} & \multirow{2}{*}{57.5} \\
\hline & R: GTGGAATGAGTCCATGCCTT & & \\
\hline \multirow{2}{*}{ P9 } & F: GACATTTCAATTCTTCTGCTTTAG & \multirow{2}{*}{ (CT) $10(\mathrm{CA}) 14$} & \multirow{2}{*}{58} \\
\hline & R: CAAGATGGCAGTGTCTTCTCA & & \\
\hline \multirow{2}{*}{ P17 } & F: TCTTGTGATCTCTTCCAGTAGAG & \multirow{2}{*}{ (TC)6cgctc(TC)9cacac(CA)14 } & \multirow{2}{*}{54} \\
\hline & R: CGTCAGGCAATGAAGGTAG & & \\
\hline \multirow{2}{*}{ P24 } & F: CAGATCCCTGAAGAATGAGG & \multirow{2}{*}{ (AC) 16} & \multirow{2}{*}{54.5} \\
\hline & R: GAGGAAGAGGATGGAGCAG & & \\
\hline \multirow{2}{*}{$\mathrm{P} 63$} & F: ATTTTCACTCCCTGCACCAA & \multirow{2}{*}{ (TA) 11} & \multirow{2}{*}{59.5} \\
\hline & R: CTCATGGGGTAAAAGGCAGA & & \\
\hline \multirow{2}{*}{ P67 } & F: CCGGGGTGCAATTAGAGTAA & \multirow{2}{*}{ (AC) 19} & \multirow{2}{*}{54.5} \\
\hline & R: GACTGCAATGGGTTTGTGTG & & \\
\hline \multirow{2}{*}{ P73 } & F: CACTGCCCAAGAGAACAAGA & \multirow{2}{*}{ (CT)6t(AC) 12} & \multirow{2}{*}{57.5} \\
\hline & R: TTTTCTGGGGTGCAAGTTTC & & \\
\hline \multirow{2}{*}{ P75 } & F: GGGAAGGAGGTTCAAGAGGA & \multirow{2}{*}{ (TG)9 } & \multirow{2}{*}{61} \\
\hline & R: CCACCATAAACTTTGTTGCCA & & \\
\hline \multirow{2}{*}{ P78 } & F: TGAATTATCCGTGTGGCAGA & \multirow{2}{*}{ (GT)7t(TG) 12 cgtgcgtgtgtgcatgtgtgtgtgcgtgt(GTGC)5 } & \multirow{2}{*}{61.5} \\
\hline & R: GTCCCTCCGTGTCTGTCTGT & & \\
\hline \multirow{2}{*}{$\mathrm{P} 90$} & F: TGCAGTGGCCATCATGTAAT & \multirow{2}{*}{ (AT) 14} & 615 \\
\hline & R: ATGTGTGCAAGTCACTTCTTTAAT & & 61.5 \\
\hline por & F: CAGTCATTCAAGACCAAGCG & (ТC)15(CA)16 & 495 \\
\hline 190 & R: CATTTTCACAAATTGAGCCCT & (IC) $13(\mathrm{CA}) 10$ & 49.3 \\
\hline P113 & F: CTGACTTCTTTCTCCCTACGA & (CT) 28 & 54 \\
\hline P110 & R: CAACCACTTTTGGATTCACAG & $(C 1) 20$ & 34 \\
\hline P154 & F: CAAGGGATCATTTCAATGCT & (AGT)9 & 585 \\
\hline P154 & R: GATACGACTGAGCGACTTGA & $(A G 1) 9$ & 58.5 \\
\hline P160 & F: AAGAGGCAGCACCGTACA & (TGC) 20 & 525 \\
\hline P160 & R: CTATGAAAGAAAGAGCCAGAGT & & 32.3 \\
\hline
\end{tabular}

Table 1. Primer pairs of 15 microsatellite loci in Tibetan antelope.

genetic consequences of intervention strategies remain unknown. Herein, we address this gap in knowledge by using 15 microsatellite loci to investigate temporal changes in the genetic diversity of Tibetan antelope during an 11-year period. The aim of this study was to examine the trends in this change and to what extent the populations have been restored at the genomic level. Our results provide guidance for future conservation and management strategies.

\section{Materials and Methods}

Materials. Skin tissue samples were obtained from Qinghai Forest Bureau in 2003, which confiscated Tibetan antelopes that were poached in the Hoh Xil National Nature Reserves (hereafter referred to as Pop2003, $\mathrm{n}=47$ ). Placental tissues were collected from the Zhuonaihu Lake area in Hoh Xil National Nature Reserve in 2013 (hereafter referred to as Pop2013, $\mathrm{n}=47$ ). All samples were washed three times with deionized water, sucked with $95 \%$ ethanol and stored at $-80^{\circ} \mathrm{C}$. All necessary approvals for collection and experimentation were acquired for the described field study from the Forestry Department of Qinghai Province, China. All procedures were in accordance with the guidelines of the regulations of experiments on animals and were approved by the China Zoological Society.

Microsatellite loci and primers. A total of 15 polymorphic microsatellite loci were screened. Seven loci (locus P1, locus P9, locus P17, locus P24, locus P113, locus P154 and locus P160) were obtained using the FIASCO method ${ }^{8}$, and another 8 loci (locus P6, locus P63, locus P67, locus P73, locus P75, locus P78, locus P90 and locus P96) were searched and developed from the Tibetan antelope genome using Perl script and MISA software (http://pgrc.ipk-gatersleben.de/misa/misa.html) for extracting the microsatellite DNA sequences from genome DNA of chiru (http://www.ncbi.nlm.nih.gov/Traces/wgs/?val=AGTT01\#contigs). The forward microsatellite primers were labeled by FAM and synthesized by Sangon Biotech (Shanghai). The primers specific for 15 microsatellite loci are shown in Table 1.

DNA extraction. Genomic DNA was extracted using the standard SDS-Phenol method ${ }^{9}$ with minor modifications. The concentration and purity of genomic DNA were measured by Nano 2000C, and template DNA was diluted to $50 \mathrm{ng} / \mu \mathrm{L}$. All loci were amplified in each of the 47 samples of Pop2003 and Pop2013. The PCR amplification was conducted in a $20-\mu \mathrm{L}$ reaction mixture, which contained $0.1 \mathrm{mmol} / \mathrm{L} \mathrm{dNTPs}, 0.2 \mu \mathrm{mol} / \mathrm{L}$ each 


\begin{tabular}{|c|c|c|c|c|c|c|c|c|}
\hline Locus & Population & NC & NA & Но & $\mathrm{He}$ & \multicolumn{2}{|c|}{ PIC } & I \\
\hline \multirow{2}{*}{ P1 } & Pop2003 & 92 & 11 & 0.804 & 0.776 & 0.750 & \multirow{2}{*}{0.774} & 1.875 \\
\hline & Pop2013 & 94 & 11 & 0.851 & 0.818 & 0.790 & & 1.968 \\
\hline \multirow{2}{*}{ P6 } & Pop2003 & 94 & 8 & 0.660 & 0.636 & 0.561 & \multirow{2}{*}{0.623} & 1.240 \\
\hline & Pop2013 & 94 & 8 & 0.702 & 0.721 & 0.669 & & 1.497 \\
\hline \multirow{2}{*}{ P9 } & Pop2003 & 94 & 14 & 0.830 & 0.840 & 0.818 & \multirow{2}{*}{0.827} & 2.165 \\
\hline & Pop2013 & 94 & 16 & 0.809 & 0.847 & 0.825 & & 2.210 \\
\hline \multirow{2}{*}{ P17 } & Pop2003 & 94 & 13 & 0.745 & 0.775 & 0.746 & \multirow{2}{*}{0.744} & 1.883 \\
\hline & Pop2013 & 90 & 9 & $0.556^{*}$ & 0.772 & 0.738 & & 1.750 \\
\hline \multirow{2}{*}{ P24 } & Pop2003 & 94 & 12 & 0.894 & 0.836 & 0.811 & \multirow{2}{*}{0.850} & 2.068 \\
\hline & Pop2013 & 94 & 13 & 0.957 & 0.892 & 0.872 & & 2.310 \\
\hline \multirow{2}{*}{ P63 } & Pop2003 & 94 & 10 & 0.851 & 0.824 & 0.792 & \multirow{2}{*}{0.820} & 1.890 \\
\hline & Pop2013 & 94 & 13 & 0.809 & 0.867 & 0.843 & & 2.170 \\
\hline \multirow{2}{*}{ P67 } & Pop2003 & 92 & 12 & 0.739 & 0.817 & 0.785 & \multirow{2}{*}{0.785} & 1.947 \\
\hline & Pop2013 & 84 & 11 & 0.643 & 0.810 & 0.771 & & 1.809 \\
\hline \multirow{2}{*}{ P73 } & Pop2002 & 94 & 11 & $0.660^{*}$ & 0.856 & 0.832 & \multirow{2}{*}{0.850} & 2.102 \\
\hline & Pop2013 & 92 & 11 & $0.196^{\star}$ & 0.866 & 0.841 & & 2.129 \\
\hline \multirow{2}{*}{ P75 } & Pop2003 & 94 & 14 & 0.957 & 0.900 & 0.880 & \multirow{2}{*}{0.896} & 2.352 \\
\hline & Pop2013 & 92 & 15 & 0.870 & 0.920 & 0.903 & & 2.524 \\
\hline \multirow{2}{*}{ P78 } & Pop2003 & 90 & 17 & 0.933 & 0.886 & 0.865 & \multirow{2}{*}{0.858} & 2.380 \\
\hline & Pop2013 & 94 & 16 & 0.809 & 0.863 & 0.840 & & 2.248 \\
\hline \multirow{2}{*}{ P90 } & Pop2003 & 94 & 14 & 0.745 & 0.842 & 0.814 & \multirow{2}{*}{0.833} & 2.075 \\
\hline & Pop2013 & 92 & 12 & 0.804 & 0.870 & 0.845 & & 2.159 \\
\hline \multirow{2}{*}{ P96 } & Pop2003 & 92 & 12 & 0.717 & 0.765 & 0.741 & \multirow{2}{*}{0.723} & 1.898 \\
\hline & Pop2013 & 94 & 12 & 0.766 & 0.726 & 0.697 & & 1.728 \\
\hline \multirow{2}{*}{ P113 } & Pop2003 & 94 & 19 & 0.957 & 0.925 & 0.909 & \multirow{2}{*}{0.913} & 2.678 \\
\hline & Pop2013 & 94 & 21 & 0.936 & 0.920 & 0.904 & & 2.719 \\
\hline \multirow{2}{*}{ P154 } & Pop2003 & 94 & 14 & $0.979^{\star}$ & 0.767 & 0.739 & 0790 & 1.911 \\
\hline & Pop2013 & 94 & 16 & 0.957 & 0.857 & 0.838 & 0.799 & 2.334 \\
\hline & Pop2003 & 94 & 11 & 0.830 & 0.867 & 0.841 & & 2.101 \\
\hline P160 & Pop2013 & 94 & 13 & 0.872 & 0.873 & 0.849 & 0.851 & 2.166 \\
\hline Meon & Pop2003 & 1 & 12.929 & 0.832 & 0.818 & & & 2.033 \\
\hline Mean & Pop2013 & I & 13.286 & 0.810 & 0.840 & & & 2.114 \\
\hline
\end{tabular}

Table 2. Genetic diversity at microsatellite loci of Pop2003 and Pop2013 of Tibetan antelope. Values of Ho marked in italic and bold indicated significant deviation $(P<0.05)$ from HWE. Population deviated from HWE after Bonferroni correction (adjusted $\alpha=0.05 / 15$ ) indicated by an asterisk. Mean values of genetic diversity parameters were calculated using 14 loci of SSR (except locus P73).

of primers, 1 Unit Taq DNA polymerase and $1 \mu \mathrm{L}$ DNA template. The PCR programme was set at $94^{\circ} \mathrm{C}$ for $2 \mathrm{~min}$, followed by 35 cycles at $94^{\circ} \mathrm{C}$ for $30 \mathrm{~s}, \mathrm{x}^{\circ} \mathrm{C}(\mathrm{x}=$ the annealing temperature specified for each set of primers, (Table 1) for $30 \mathrm{~s}$ and $72^{\circ} \mathrm{C}$ for $30 \mathrm{~s}$. A final step of $10 \mathrm{~min}$ at $72^{\circ} \mathrm{C}$ completed the programme. GeneScanTM-500 LIZTM was used as internal standard for Polymorphism detection on a ABI 3730 DNA Sequencer (Applied Biosystems, Inc.). GeneMarker V1.75 (Applied Biosystems, Inc.) was used for genotype interpretation.

Statistical analysis of genetic diversity parameters. Original data were analyzed and manually corrected to validate the accurate peak shape and allele size using GeneMarker V1.75. Micro-Checker was used to validate the availability of genotype data ${ }^{10}$. The transformations for genotype data formats were conducted by Convert V1.3.1 $1^{11}$ for subsequent analysis. Linkage disequilibrium (LD) test was analyzed by Genepop V4.4 $4^{12,13}$. Number of Alleles (NA) and Shannon index (I) were calculated by Microsatellite Analyzer V4.05 ${ }^{14}$. We calculated observed heterozygosity $(\mathrm{Ho})$, expected heterozygosity $(\mathrm{He})$ and analyzed the Hardy-Weinberg equilibrium (HWE) by implementing Arlequin V3.5 ${ }^{15}$. Polymorphism information content (PIC) was estimated by modified PowerStats Worksheet ${ }^{16}$. BOTTLENECK V1.2.02 ${ }^{17,18}$ was used to validate whether the population had undergone the bottleneck effect. Under a two-phase model (TPM), we constrained the model by defining $90 \%$ of mutations as conforming to a stepwise mutation model and $10 \%$ as multi-step. Furthermore, the change in Ne was estimated under the infinite allele model according to the formula $\Theta$ (theta) $=4 \mathrm{Ne} \times \mathrm{Mu}$, where $\mathrm{Ne}$ is the effective population size and $\mathrm{Mu}$ is the microsatellite mutation ratio.

\section{Results}

Hardy-Weinberg equilibrium. For the 15 polymorphic loci surveyed, a total of 223 alleles were observed in both Pop2003 and Pop2013, 192 alleles were found in Pop2003, and 197 alleles were found in Pop2013. Overall microsatellite variability was high (8-19 alleles per locus) in Pop2003, and NA varied from 8 to 21 in Pop2013 (Table 2). Among all of the 15 loci, only 8 alleles at locus P6 were detected in Pop2003 or Pop2013, while the 


\begin{tabular}{|c|c|c|c|c|}
\hline Population & Test & IAM & TPM & SMM \\
\hline \multirow{3}{*}{ Pop2003 } & $\begin{array}{c}\text { Sign test: No. of loci with } \\
\text { heterozygosity excess (probability) }\end{array}$ & $7.940(\mathrm{p}=0.004)$ & $8.240(\mathrm{p}=0.339)$ & $8.300(\mathrm{p}=0.065)$ \\
\hline & $\begin{array}{c}\text { Standardized differences test: T2 } \\
\text { values (probability) }\end{array}$ & $2.711(\mathrm{p}=0.003)$ & $-0.35(\mathrm{p}=0.363)$ & $-1.609(\mathrm{p}=0.054)$ \\
\hline & $\begin{array}{l}\text { Wilcoxon test (Probability of } \\
\text { heterozygote excess) }\end{array}$ & 0.001 & 0.665 & 0.923 \\
\hline \multirow{3}{*}{ Pop2013 } & $\begin{array}{c}\text { Sign test: No. of loci with } \\
\text { heterozygosity excess (probability) }\end{array}$ & $7.990(\mathrm{p}=0.024)$ & $8.260(\mathrm{p}=0.681)$ & $8.310(\mathrm{p}=0.000)$ \\
\hline & $\begin{array}{c}\text { Standardized differences test: T2 } \\
\text { values (probability) }\end{array}$ & $2.418(\mathrm{p}=0.008)$ & $-0.934(\mathrm{p}=0.175)$ & $-2.343(\mathrm{p}=0.010)$ \\
\hline & $\begin{array}{l}\text { Wilcoxon test (Probability of } \\
\text { heterozygote excess) }\end{array}$ & 0.000 & 0.805 & 0.985 \\
\hline
\end{tabular}

Table 3. Results (P-values) of bottleneck testing under three models. Analyses with BOTTLENECK used three microsatellite mutation models: infinite allele model (IAM), two-phase model (TPM) and stepwise mutation model (SMM).

maximum number of alleles was found at locus P113 (19 and 21 alleles in Pop2003 and Pop2013, respectively). At each locus, the frequencies differed significantly among alleles. The lowest allele frequency was 1 , accounting for $1.1 \%$ of all alleles, but the highest frequency was 46 , accounting for $48.9 \%$ of all alleles. HWE test showed significant deviations from HWE at locus P17, locus P67, locus P73 and locus P154 $(P<0.001)$. However, significant deviations from HWE were confirmed only at locus P73 and locus P154 in Pop2003 and locus P17 and locus P73 in Pop2013 after Bonferroni correction (adjusted $\alpha=0.05 / 15$ ).

Linkage disequilibrium test. At locus P73, significant LD was observed in both Pop2003 and Pop2013 $(P<0.05)$. We therefore excluded this locus in subsequent analysis. Over 14 microsatellite loci, microsatellite loci pairs P1/P9, P9/P78 and P24/P96 were in LD $(P<0.05)$. However, no LD was detected between these locus pairs after Bonferroni corrections (adjusted $\alpha=0.05 / 14$ ). In Pop2013, there was significant LD between 55 locus pairs (between locus P1 and P9, P24, P75, P78, P96, P113, P154, P160; locus P6 and P9, P75, P113, P160; locus P9 and P24, P75, P78, P90, P96, P113, P154, P160; locus P17 and P24, P75, P113; locus P24 and P63, P67, P75, P78, P96, P113, P154, P160; locus P63 and P67, P75, P96, P113, P154, P160; locus P67 and P75, P113, P154; locus P75 and P78, P96, P113, P154, P160; locus P75 and P78 and P96, P113, P160; locus P78 and P96, P113, P154, P160; locus P154 and P160). However, only 22 locus pairs were found to be in significant LD after applying Bonferroni corrections for multiple tests (Table S1). Our results suggested that these microsatellite loci were relatively independent in the Tibetan antelope.

Genetic diversity. Microsatellite data of 14 microsatellite loci revealed abundant genetic diversity in both Pop2013 and Pop2003 (Table 1). In Pop2003, the mean number of alleles (MNA) was 12.92 (range 8-19), the average $H o$ was 0.832 (range $0.660-0.979$ ), and the average $H e$ was 0.810 (range 0.636-0.925). In Pop2013, the average of $\mathrm{NA}, \mathrm{Ho}$ and $\mathrm{He}$ across 14 loci was $13.133,0.810$ and 0.840 , respectively; $N A$ varied from 8 to $21, \mathrm{Ho}$ varied from 0.556 to 0.957 and $H e$ varied from 0.721 to 0.920 . The PIC at each microsatellite locus was always larger than 0.5 (range 0.561 to 0.909 ), a threshold value considered to be highly informative. Shannon information index ranged from 1.240 to 2.678 , which also indicated a high genetic diversity.

Bottleneck effect. The infinite allele model (IAM), TPM and stepwise mutation model (SMM) were applied when BOTTLENECK was used to test for population bottlenecks in this study. Populations exhibiting a significant heterozygosity excess would be considered to have experienced a recent genetic bottleneck. Under the TPM, the results displayed no genetic bottleneck effect in either Pop2003 or Pop2013 (Table 3). Furthermore, the Wilcoxon test, which is considered to be more reliable than the sign test and standardized differences test, showed no significant results for population bottleneck under the SMM (Pop2003: $P=0.923$; Pop2013: $P=0.985$ ). The sign test, standardized differences test and Wilcoxon test all showed a significant heterozygosity excess in either Pop2003 or Pop2013 under the IAM $(P<0.05)$. However, this was not necessarily indicative of true heterozygosity excess, as the IAM is thought to be a less appropriate model for microsatellites than the $\mathrm{SMM}^{19}$. These results thus point to the population resilience of the Tibetan antelope.

Recovery of genetic variation. A tendency toward recovery of genetic diversity was observed in the Tibetan antelope. Pop2013, compared with Pop2003, exhibited higher values in He, PIC, I and $\Theta$ at 9 out of 14 loci (locus P1, P6, P9, P24, P63, P75, P90, P154, P160). In comparison with Pop2003, paired t-test demonstrated that Pop2013 had significantly higher values of average $H e(0.840 \mathrm{Vs} 0.818, P<0.05)$ and $P I C(0.813 \mathrm{Vs} 0.789$, $P<0.05)$ but differences in average $M N A(13.133 \mathrm{Vs} 12.929, P>0.05)$ and $H o(2.144 \mathrm{Vs} 2.033, P>0.05)$ were not found (Table 2). There was also a near significant rise $(\mathrm{P}=0.060)$ in $I$, indicating genetic recovery in the Tibetan antelope. Furthermore, the average $\Theta$ increased from 4.581 (Pop2003) to 5.305 (Pop2013) over an 11-year period (Table 4), showing that the population size had increased significantly over approximately a decade $(P<0.05)$. 


\begin{tabular}{|l|c|c|c|c|}
\hline Locus & Pop2003 & Pop2013 & Mean & s.d. \\
\hline P1 & 3.467 & 4.484 & 3.976 & 0.719 \\
\hline P6 & 1.744 & 2.586 & 2.165 & 0.595 \\
\hline P9 & 5.262 & 5.553 & 5.408 & 0.206 \\
\hline P17 & 3.438 & 3.387 & 3.412 & 0.036 \\
\hline P24 & 5.088 & 8.261 & 6.674 & 2.244 \\
\hline P63 & 4.691 & 6.536 & 5.614 & 1.304 \\
\hline P67 & 4.479 & 4.250 & 4.365 & 0.162 \\
\hline P75 & 8.957 & 11.533 & 10.245 & 1.822 \\
\hline P78 & 7.783 & 6.285 & 7.034 & 1.059 \\
\hline P90 & 5.344 & 6.681 & 6.012 & 0.945 \\
\hline P96 & 3.250 & 2.649 & 2.949 & 0.425 \\
\hline P113 & 12.326 & 11.489 & 11.907 & 0.592 \\
\hline P154 & 3.298 & 5.971 & 4.635 & 1.890 \\
\hline P160 & 6.497 & 6.847 & 6.672 & 0.247 \\
\hline Mean & 4.503 & 5.235 & 4.869 & 0.518 \\
\hline
\end{tabular}

Table 4. Estimation of theta $(\theta)$ in Pop2003 and Pop2013 of Tibetan antelope.

\begin{tabular}{|l|c|c|c|}
\hline Species & Researcher & MNA & He \\
\hline Banteng Bos javanicus & 40 & 2.42 & 0.47 \\
\hline Wild gaur B. gaurus & 41 & 2.2 & $0.091-0.835$ \\
\hline Yak B. grunniens & 42 & 11.69 & 0.616 \\
\hline \multirow{2}{*}{ Przewalski's gazelle Procapra przewalskii } & 43 & 5.98 & 0.780 \\
\cline { 2 - 4 } & 44 & 5.85 & 0.552 \\
\hline American Bison Bison bison & 45 & $3.56-5.00$ & $0.522-0.652$ \\
\hline Dorcas gazelle Gazella Dorcas & 46 & $2.2-7.0$ & $0.466-0.727$ \\
\hline African wild ass Equus africanus & 47 & 5.06 & 0.59 \\
\hline Huemul Hippocamelus bisulcus & 48 & 5.25 & 0.461 \\
\hline \multirow{2}{*}{ Chiru Pantholops hodgsoni Pop2003/Pop2013 } & 20 & 9.4 & 0.838 \\
\cline { 2 - 4 } & This study & $12.800 / 13.133$ & $0.821 / 0.841$ \\
\hline
\end{tabular}

Table 5. Summary of genetic diversity parameters of microsatellite data of several Bovidae species.

\section{Discussions}

Genetic diversity. We examined 15 microsatellite loci in this study to assess the genetic variation in the Tibetan antelope. Our data show rich genetic diversity in Pop2003 and Pop2013 with high values in MNA, He and PIC. Although the results are not directly comparable because different microsatellite loci were used, comparisons of the Tibetan antelope to other Bovidae species suggest that genetic diversity in the Tibetan antelope ranks highest (Table 5), with values of 12.929/13.286 and 0.818/0.840 for MNA and He in Pop2003 and Pop2013, respectively. Compared to the domestic yak, which is herded in the QTP and the adjacent Asian highlands with a population of more than 14 million, the Tibetan antelope exhibits higher genetic variation (Table 5). These results are in agreement with a previous genetic analysis ${ }^{20}$ and our previous study ${ }^{21}$, in which significant heterogeneity in the frequencies of mtDNA control region haplotypes was observed.

Bottleneck signature detection. Bottleneck detection is critical for interpreting the historical demography of populations and is informative for establishing conservation strategies for endangered animals. Simulations inferred from mtDNA D-loop fragment show that the Tibetan antelope experienced a severe historical demographic decline since approximately five thousand years before present (B.P.) ${ }^{21}$. Although the Wilcoxon test detected no significant recent population bottleneck signature in the Tibetan antelope under the TPM and SMM via BOTTLENECK in the present study, a recent well-documented decline in the population size of the Tibetan antelope has occurred over the last century, with population size decreasing from a maximum of approximately 500,000-1,000,000 to a minimum of 50,000 2,3 . Heavy illegal poaching associated with a profitable fur trade could account for this severe demographic reduction. The bottleneck was not detected using the Wilcoxon test for heterozygous excess probably because the number of loci analyzed was small ${ }^{17,18}$ or due to an insufficient sample size ${ }^{17}$.

Slow post-bottleneck recovery of genetic diversity. Demographic bottlenecks can result in a loss of genetic variation ${ }^{22-26}$ due to the bottleneck effect and subsequent genetic drift, as has already been observed in African elephants ${ }^{27}$, black-footed ferrets ${ }^{28}$ and Arctic foxes ${ }^{29}$. Rather than rapid genetic loss, the results presented 
here suggest a slow post-bottleneck recovery of genetic diversity (in terms of both allele numbers and heterozygosity) in the Pop2013 population in comparison to the Pop2003 population, with values from 12.800 to 13.133 and from 0.821 to 0.841 for $M N A$ and $H e$, respectively.

Studies have indicated that factors such as dispersive capabilities ${ }^{30-34}$ and effective population size ${ }^{35-37}$ may affect the change in genetic variation. High dispersal potential due to migration of females in most populations each year to summer calving ground $s^{38,39}$ is assumed to promote frequent gene flow. Substantial gene flow was detected in our earlier investigation by examining mtDNA fragments ${ }^{21}$. Therefore, recovery of genetic variation via gene flow is expected, especially within the large populations. Moreover, starting in the 1990s, the Chinese government established seven Nature Reserves and constructed corridors for facilitating the migration of Tibetan antelope, both of which have likely facilitated gene flow among populations and reduced genetic loss in post-bottleneck populations.

We conclude that ample genetic diversity may still exist in the Tibetan antelope. Furthermore, the Tibetan antelope has shown a slight increase in genetic variation during the past 11-year period. In this sense, the results of the present study suggest that protection efforts did not arrive too late for the Tibetan antelope and provide molecular evidence for the effectiveness of conservation strategies.

\section{References}

1. Schaller, G. B. Wildlife of the Tibetan steppe. (University of Chicago Press, 1998).

2. Xia, L. \& Yang, Q. S. Migration and protection of Tibetan antelope. Bulletin of Biology 50, 12-15 (2015).

3. Leclerc, C., Bellard, C., Luque, G. M. \& Courchamp, F. Overcoming extinction: understanding processes of recovery of the Tibetan antelope. Ecosphere 6, doi: Artn 171 10.1890/Es15-00049.1 (2015).

4. Wu, X. M. \& Zhang, H. F. Resources regarding populations of Tibetan Antelope (Pantholops hodgsoni) ant the status of its protection. Chinese Journal of Nature 33, 143-148 (2011).

5. Zhang, B. et al. Genetic viability and population history of the giant panda, putting an end to the "evolutionary dead end"? Molecular biology and evolution 24, 1801-1810, doi: 10.1093/molbev/msm099 (2007).

6. Kvist, L. et al. Population decline is accompanied by loss of genetic diversity in the Lesser Grey Shrike Lanius minor. Ibis 153, 98-109, doi: 10.1111/j.1474-919X.2010.01091.x (2011).

7. Chang, Z. F. et al. Human influence on the population decline and loss of genetic diversity in a small and isolated population of Sichuan snub-nosed monkeys (Rhinopithecus roxellana). Genetica 140, 105-114, doi: 10.1007/s10709-012-9662-9 (2012).

8. Zane, L., Bargelloni, L. \& Patarnello, T. Strategies for microsatellite isolation: a review. Molecular ecology 11, 1-16, doi: DOI 10.1046/j.0962-1083.2001.01418.x (2002).

9. Sambrook, J. \& Russell, D. (Cold Spring Harbor Laboratory Press, Cold Spring Harbor, New York, 2001).

10. Van Oosterhout, C., Hutchinson, W. F., Wills, D. P. \& Shipley, P. MICRO-CHECKER: software for identifying and correcting genotyping errors in microsatellite data. Molecular Ecology Notes 4, 535-538 (2004).

11. Glaubitz, J. C. CONVERT: A user-friendly program to reformat diploid genotypic data for commonly used population genetic software packages. Molecular Ecology Notes 4, 309-310, doi: DOI 10.1111/j.1471-8286.2004.00597.x (2004).

12. Raymond, M. \& Rousset, F. Genepop (Version-1.2)-Population-Genetics Software for Exact Tests and Ecumenicism. Journal of Heredity 86, 248-249 (1995).

13. Rousset, F. genepop'007: a complete re-implementation of the genepop software for Windows and Linux. Molecular ecology resources 8, 103-106 (2008).

14. Dieringer, D. \& Schlotterer, C. MICROSATELLITE ANALYSER (MSA): a platform independent analysis tool for large microsatellite data sets. Molecular Ecology Notes 3, 167-169, doi: 10.1046/j.1471-8286.2003.00351.x (2003).

15. Excoffier, L. \& Lischer, H. E. L. Arlequin suite ver 3.5: a new series of programs to perform population genetics analyses under Linux and Windows. Molecular Ecology Resources 10, 564-567, doi: 10.1111/j.1755-0998.2010.02847.x (2010).

16. Tereba, A. Tools for analysis of population statistics. Profiles in DNA 2, 14-16 (1999).

17. Cornuet, J. M. \& Luikart, G. Description and power analysis of two tests for detecting recent population bottlenecks from allele frequency data. Genetics 144, 2001-2014 (1996).

18. Piry, S., Luikart, G. \& Cornuet, J.-M. BOTTLENECK: a program for detecting recent effective population size reductions from allele data frequencies. Journal of Heredity 90, 502-503 (1999).

19. Shriver, M. D., Jin, L., Chakraborty, R. \& Boerwinkle, E. VNTR allele frequency distributions under the stepwise mutation model: a computer simulation approach. Genetics 134, 983-993 (1993).

20. Zhou, H., Li, D. Q., Zhang, Y. G., Yang, T. \& Liu, Y. Genetic diversity of microsatellite DNA loci of Tibetan antelope (Chiru, Pantholops hodgsonii) in Hoh Xil National Nature Reserve, Qinghai, China. J Genet Genomics 34, 600-607, doi: 10.1016/S16738527(07)60068-X (2007).

21. Du, Y. R. et al. Demographic history of the Tibetan antelope Pantholops hodgsoni (chiru). J Syst Evol 48, 490-496, doi: 10.1111/j.1759-6831.2010.00095.x (2010).

22. Bouzat, J. L., Paige, K. N. \& Lewin, H. A. The ghost of genetic diversity past: historical DNA analysis of the greater prairie chicken. The American Naturalist 152, 1-6 (1998).

23. Chakraborty, R. \& Nei, M. Bottleneck effects on average heterozygosity and genetic distance with the stepwise mutation model. Evolution, 347-356 (1977).

24. Lacy, R. C. Loss of genetic diversity from managed populations: interacting effects of drift, mutation, immigration, selection, and population subdivision. Conservation Biology 1, 143-158 (1987).

25. Nei, M., Maruyama, T. \& Chakraborty, R. Bottleneck Effect and Genetic-Variability in Populations. Evolution 29, 1-10, doi: Doi $10.2307 / 2407137$ (1975).

26. Wright, S. Evolution and the genetics of populations: Vol. 2. The theory of gene frequencies. (1969).

27. Whitehouse, A. M. \& Harley, E. H. Post-bottleneck genetic diversity of elephant populations in South Africa, revealed using microsatellite analysis. Molecular ecology 10, 2139-2149, doi: DOI 10.1046/j.0962-1083.2001.01356.x (2001).

28. Wisely, S. M., Buskirk, S. W., Fleming, M. A., McDonald, D. B. \& Ostrander, E. A. Genetic diversity and fitness in black-footed ferrets before and during a bottleneck. Journal of Heredity 93, 231-237, doi: DOI 10.1093/jhered/93.4.231 (2002).

29. Ploshnitsa, A. I., Goltsman, M. E., Happ, G. M., Macdonald, D. W. \& Kennedy, L. J. Historical and modern neutral genetic variability in Mednyi Arctic foxes passed through a severe bottleneck. J Zool 289, 68-76, doi: 10.1111/j.1469-7998.2012.00964.x (2013).

30. Wenink, P. W., Groen, A. F., Roelke-Parker, M. E. \& Prins, H. H. T. African buffalo maintain high genetic diversity in the major histocompatibility complex in spite of historically known population bottlenecks. Molecular ecology 7, 1315-1322, doi: 10.1046/j.1365-294x.1998.00463.x (1998).

31. Congiu, L. et al. Managing polyploidy in ex situ conservation genetics: the case of the critically endangered Adriatic sturgeon (Acipenser naccarii). PloS one 6, e18249, doi: 10.1371/journal.pone.0018249 (2011) 
32. McEachern, M. B., Van Vuren, D. H., Floyd, C. H., May, B. \& Eadie, J. M. Bottlenecks and rescue effects in a fluctuating population of golden-mantled ground squirrels (Spermophilus lateralis). Conserv Genet 12, 285-296, doi: 10.1007/s10592-010-0139-z (2011).

33. Vandewoestijne, S., Schtickzelle, N. \& Baguette, M. Positive correlation between genetic diversity and fitness in a large, wellconnected metapopulation. Bmc Biol 6, doi: Artn 46 10.1186/1741-7007-6-46 (2008).

34. Vila, C. et al. Rescue of a severely bottlenecked wolf (Canis lupus) population by a single immigrant. Proceedings. Biological sciences/ The Royal Society 270, 91-97, doi: 10.1098/rspb.2002.2184 (2003).

35. Allendorf, F. W. \& Phelps, S. R. Loss of Genetic-Variation in a Hatchery Stock of Cutthroat Trout. T Am Fish Soc 109, 537-543, doi: 10.1577/1548-8659(1980)109<537:Logvia >2.0.Co;2 (1980).

36. Frankham, R. Relationship of genetic variation to population size in wildlife. Conservation Biology 10, 1500-1508, doi: 10.1046/j.1523-1739.1996.10061500.x (1996).

37. Reed, D. H. \& Frankham, R. Correlation between fitness and genetic diversity. Conservation Biology 17, 230-237, doi: 10.1046/j.1523-1739.2003.01236.x (2003).

38. Bleisch, W. V. et al. Surveys at a Tibetan antelope Pantholops hodgsonii calving ground adjacent to the Arjinshan Nature Reserve, Xinjiang, China: decline and recovery of a population. Oryx 43, 191-196, doi: 10.1017/S0030605308001506 (2009).

39. Buzzard, P. J., Wong, H. M. \& Zhang, H. B. Population increase at a calving ground of the Endangered Tibetan antelope Pantholops hodgsonii in Xinjiang, China. Oryx 46, 266-268, doi: 10.1017/S0030605311001657 (2012).

40. Nijman, I. J. et al. Hybridization of banteng (Bos javanicus) and zebu (Bos indicus) revealed by mitochondrial DNA, satellite DNA, AFLP and microsatellites. Heredity 90, 10-16, doi: 10.1038/s..hdy.6800174 (2003).

41. Nguyen, T. T. et al. Genomic conservation of cattle microsatellite loci in wild gaur (Bos gaurus) and current genetic status of this species in Vietnam. BMC genetics 8, doi: Artn 77 10.1186/1471-2156-8-77 (2007).

42. Zhang, G. X. et al. Analysis of genetic diversity and population structure of Chinese yak breeds (Bos grunniens) using microsatellite markers. J Genet Genomics 35, 233-238, doi: Doi 10.1016/S1673-8527(08)60032-6 (2008).

43. Hong, Y., Li, D., Yi, T., Zhang, Y. \& Liu, Y. Screening Microsatellite Primers and their Application for Individual Identification in Faecal DNA from Procapra praprzewalskii. Chinese Agricultural Science Bulletin 9, 015 (2008).

44. Yang, J. \& Jiang, Z. G. Genetic diversity, population genetic structure and demographic history of Przewalski's gazelle (Procapra przewalskii): implications for conservation. Conserv Genet 12, 1457-1468, doi: 10.1007/s10592-011-0244-7 (2011).

45. Hedrick, P. W. Conservation genetics and North American bison (Bison bison). The Journal of heredity 100, 411-420, doi: 10.1093/ jhered/esp024 (2009).

46. Godinho, R. et al. Conservation genetics of the endangered Dorcas gazelle (Gazella dorcas spp.) in Northwestern Africa. Conserv Genet 13, 1003-1015, doi: 10.1007/s10592-012-0348-8 (2012).

47. Rosenbom, S., Costa, V., Steck, B., Moehlman, P. \& Beja-Pereira, A. Cross-species genetic markers: a useful tool to study the world's most threatened wild equid-Equus africanus. Eur J Wildlife Res 58, 609-613, doi: 10.1007/s10344-011-0578-y (2012).

48. Marin, J. C. et al. Cross-amplification of nonspecific microsatellites markers: a useful tool to study endangered/vulnerable species of southern Andes deer. Genetics and Molecular Research 13, 3193-3200, doi: 10.4238/2014.April.25.4 (2014)

\section{Acknowledgements}

This study was funded by the Promoting Innovation Ability Program on Science \& Technology in Qinghai province of China (2013-Z-751 and 2013-Z-750) and the CAS "Light of West China" Program. We are also grateful to Dr. Qi-En Yang for his helpful reading of the manuscript and constructive comments.

\section{Author Contributions}

S.G. and J.M. conceived and designed the research; X.Z., Y.X., X.G., S.L., X.Z.Z. and M.S. collected the materials and performed the experiments; S.G. and Y.D. analyzed the data; and S.G., J.B.M. and Y.D. wrote the paper. All authors contributed to revision and reviewed the manuscript.

\section{Additional Information}

Supplementary information accompanies this paper at http://www.nature.com/srep

Competing financial interests: The authors declare no competing financial interests.

How to cite this article: Du, Y. et al. Microsatellite Loci Analysis Reveals Post-bottleneck Recovery of Genetic Diversity in the Tibetan Antelope. Sci. Rep. 6, 35501; doi: 10.1038/srep35501 (2016).

(c) (i) This work is licensed under a Creative Commons Attribution 4.0 International License. The images or other third party material in this article are included in the article's Creative Commons license, unless indicated otherwise in the credit line; if the material is not included under the Creative Commons license, users will need to obtain permission from the license holder to reproduce the material. To view a copy of this license, visit http://creativecommons.org/licenses/by/4.0/

(c) The Author(s) 2016 